\title{
MiR-493-5p inhibits Th9 cell differentiation in Allergic Asthma by targeting FOXO1
}

\author{
Weili Zhang ${ }^{1}$, Gang Li $^{1}$, He-ting Dong ${ }^{1}$, Hui-ming Sun ${ }^{1}$, Wen-jing Gu${ }^{1}$, Xin-xing Zhang ${ }^{1}$, \\ Mei-juan Wang ${ }^{1}$, Li Huang ${ }^{1}$, Yong-dong Yan $^{1}$, Chuang-li Hao ${ }^{1}$, Wei $\mathrm{Ji}^{1}$, Can-hong Zhu ${ }^{1}$, \\ and Zhengrong Chen ${ }^{1}$ \\ ${ }^{1}$ Children's Hospital of Soochow University
}

May 5, 2021

\begin{abstract}
Background:There is an increasing attention on miRNAs because of their functional effect on polarization of CD4+ T cells. This study explores the mechanism of miR-493-5p regulating Th9 cell differentiation in allergic asthma. Methods: The allergic airway inflammation is induced by Ovalbumin(OVA)in mice. CD4+ T cells from normal mice are cultured under Th9 cell conditions. IL-9 levels in mice and CD4+T cells are analyzed by RT-qPCR, ELISA, flow cytometry and western blot. The miR-493-5p levels in mice and cells are detected by RT-qPCR. The interaction between FOXO1 and miR-493-5p is predicted by TargetScan and confirmed by dual luciferase assay. The pathological state is evaluated by H\&E staining and Lung resistance is measured in the allergic mice treated with miR-493-5p agomiR before stimulation. Results: The miR-493-5p expression in OVA-induced mice decreases significantly, accompanied by a significant upregulation in IL-9, IRF4 and FOXO1 expression and proportion of CD4+Th9 cells. MiR-493-5p mimic inhibits the expression of IL-9, IRF4 and FOXO1 and Th9 cell differentiation, while the inhibitor promotes these effects. MiR-493-5p mimic represses FOXO1 expression through interacting with 3'UTR of FOXO1 mRNA. The rescue experiment proves that miR-493-5p regulates the differentiation of Th9 cell and the expression of IL-9 by targeting FOXO1. In addition, we find that miR-493-5p agomiR treatment inhibits the FOXO1, IL-9 and IRF4 expression, decreases the proportion of CD4+ Th9 cells, alleviates the pathological state of lung tissue and airway hyperreactivity in OVAinduced asthma mice. Conclusions: Our study confirmed that miR-493-5p inhibited Th9 cell differentiation in allergic asthma by targeting FOXO1.
\end{abstract}

\section{INTRODUCTION}

Asthma is defined by the history of respiratory symptoms such as wheeze, shortness of breath, chest tightness and cough that vary over time and in intensity, together with variable expiratory airflow limitation(1). It is the most common chronic disease of childhood and the leading cause of childhood morbidity from chronic disease(2), but its pathogenesis is still not fully elucidated.

Allergic asthma is the most common phenotype, associated with type 2 cytokines interleukin-4(IL-4), IL-5, IL-9 and IL-13, which promote airway eosinophils, mucus overproduction, bronchial hyperresponsiveness, and immunoglobulin E synthesis(. Among them, IL-9 was reported as an active participant in the pathogenesis of allergic asthma(4). Jia et al(have found that IL-9 level was significantly elevated in allergic asthma children, along with the existence of antigen-specific Th9 cells, suggesting that Th9 cells may be the major source of IL-9 in children with allergic asthma. As a helper T lymphocyte subpopulation, Th9 cells differentiates from Th2 cells, is a more specialized IL-9-producing cell and has been shown to be pro-inflammatory in vivo)due to the production of IL-9. Recently, several studies have reported that Th9/IL-9 is associated with asthma(8, 9). Therefore, inhibition of Th9 cells differentiation may be a potential immunotherapy for asthma. 
MicroRNAs(miRNAs)are well-known short non-coding RNAs that regulate gene expression by complementary base pairing with the 3'-untranslated region(3' UTR)of mRNAs(10). There is an increasing attention on miRNAs because of their functional effect on polarization of $\mathrm{CD}^{+} \mathrm{T}$ cells. Through high-throughput sequencing, we found that there were many abnormal miRNAs in PBMCs of asthmatic children compared with normal children. Further gene ontology(GO)analysis and Kyoto Encyclopedia of Genes and Genomes(KEEG)signaling pathway analysis showed that the significantly down regulated miR-493-5p in PBMCs of asthmatic children was related to cell differentiation. In addition, the transcription factor forkhead box O1(FOXO1)was reported to be a positive regulator of Th9 cell differentiation by binding to promoters of IL-9 and IRF4(11). And FOXO1 was a target gene of miR-493-5p predicted by TargetScan.

Therefore, based on the above reports and prediction results, we speculated that miR-493-5p might inhibit Th9 cell differentiation by targeting FOXO1, thereby reducing IL-9 level and participating in the regulation of inflammatory response in asthma.

\section{MATERIALS AND METHODS}

\section{High Throughput Sequencing}

PBMCs were isolated from peripheral blood collected from asthmatic children $(\mathrm{n}=5)$ and normal children $(n=5)$. We used High throughput sequencing to screen different expression of miRNAs. GO and KEEG signal pathway analysis were launched to show the function of miRNAs, and we selected the satisfactory miRNA.

\section{Animals}

4 5-weeks-old male mice BALB/c were obtained from the Specific Pathogen-Free Laboratory Animal Centre of Soochow University. All animal procedures were conducted in accordance with the guidelines of the Institutional Animal Care and Use Committee, Soochow University Research Institute of Bioscience and Biotechnology. They were divided into several groups according to the experimental needs $(\mathrm{n}=8)$. All mice were fed adaptively for one week before the experiment.

\section{Murine Asthma Model and MiR-493-5p AgomiR Treatment}

$428 \mu \mathrm{g}$ OVA(Sigma, SLBP0782V)was dissolved in 300 $\mu \mathrm{L}$ PBS containing 3mg aluminum hydroxide(Sinopharm)as the amount of injection for each mouse. After the preparation of OVA suspension, 200ul was injected into the groin and abdominal cavity on both sides of the mice, once a week for 2 weeks. Following that, stimulation with intranasal administration of OVA was given continuously for 7 days to construct the asthma model. Three days before the stimulation, one dose of miR-493-5p agomiR or agomiR NC was delivered by trachea. After stimulation, the behavior of mice in the asthma group was observed. In the process of intranasal administration, the behavior of mice in the asthma group gradually appeared restlessness, forelimb retraction and lifting. Meantime, there were panting phenotypes such as frequent nodding breath, shortness of breath and incontinence of two stools, which indicated that the asthma model was established successfully.

\section{Isolation of $\mathrm{CD}^{+} \mathrm{T}$ Cells}

The peripheral blood of normal mice was collected and the PBMC cells were obtained by density gradient centrifugation, and the $\mathrm{CD} 4^{+} \mathrm{T}$ cells were separated by magnetic beads according to the instructions.

\section{Induction of Th9 Cells}

$\mathrm{CD} 4^{+} \mathrm{T}$ cells isolated from the peripheral blood of normal mice was added with $3 \mathrm{ml}$ Th9 induction medium(10ng/mL IL-4(PeproTech), 3ng/mL TGF- $\beta$ (GenScript)and 10ng/mL IFN- $\gamma$ (PeproTech)for seven days, and the medium was changed every three days.

\section{$\mathrm{CD}^{+} \mathrm{T}$ Cells Transfection}


The miR-493-5p mimic/inhibitor, FOXO1 interfere sequences(siRNA)and their negative control(NC)were synthesized, and the FOXO1 overexpressing plasmids and empty control were purchased from Suzhou GenePharma Co., Ltd. The plasmids were diluted and mixed with serum-free medium according to the instructions. Then it was mixed with Lipofectamine 2000(Invitrogen)diluted with serum-free medium and incubated at room temperature for $5 \mathrm{~min}$, and then incubated at room temperature for $20 \mathrm{~min}$ to form the complex. Then the $\mathrm{CD} 4^{+} \mathrm{T}$ cells were co-cultivated with the complex at 37 in $5 \% \mathrm{CO}_{2}$ incubator for $6 \mathrm{~h}$.

\section{ELISA}

Bronchoalveolar lavage fluid(BALF)was obtained by recovering the irrigation solution and centrifugation at 2000rpm for 10min. Lung tissue was collected, lavaged and homogenized. The supernatant was also collected. The IL-9 levels in lung tissues, BALF and supernatant were tested by ELISA with IL-9 kit(Elabscience,EEL-M0724c)according to the instructions, and the OD value of each blank was measured by luminescent detector(Molecular Devices SpectraMax@i3 ) under 450nm wavelength light.

\section{RNA Extraction and RT-qPCR analysis}

Total RNA of lung tissue and cells were isolated using TRIzol(Invitrogen)according to the manufacturer's protocol. Reverse transcription of mature mRNAs was performed using $1 \mu \mathrm{g}$ mRNA, AMV Reverse Transcriptase(Thermo \#K1622), RNase inhibitor and dNTP. Reverse transcription of FOXO1, IRF4, IL-9 were performed using the SYBR Green qPCR kit(Thermo F-415XL)according to the manufacturer's protocol. U6 and $\beta$-actin were used as internal controls for miR-493-5p and FOXO1, IRF4 and IL-9 respectively. The primer sequences were listed in Table 1 . The $2^{-[?][?] \mathrm{Ct}}$ method was used to quantify the expression.

\section{Flow Cytometry}

Cells were adjusted to $10^{6}$ cells $/ \mathrm{mL}$ and cultivated with $2 \mu \mathrm{L}$ monensin for $6 \mathrm{~h}$, and then washed with $1 \times$ perm/wash buffer and centrifuged. 100 $\mu \mathrm{L}$ of staging buffer and $0.5 \mu \mathrm{L}$ of CD4 antibody(Biolegend)was mixed into each pore, and then incubated in a refrigerator at 4 in dark for $30 \mathrm{~min}$. Cells were washed twice with $1 \times$ perm/wash buffer, and $250 \mu \mathrm{L}$ fixation/permeabilization solution(with BD GolgiStopTM)was injected into each hole, then the cells were incubated in refrigerator at 4 for $20 \mathrm{~min}$. The cells were washed with $1 \times$ perm/wash buffer and then centrifugated. $0.5 \mu \mathrm{L}$ of IL-9 antibody(BioLegend)was mixed with 100 $\mu \mathrm{L}$ of Staining Bufferin to each pore. Incubated in a refrigerator at 4 in dark for 30min, the cells were washed twice with $1 \times$ perm/wash buffer, and then resuspended with Staining Buffer. The quantitative detection was performed immediately by flow cytometry(BD-FACSVerse).

\section{Dual Luciferase Assay}

The FOXO1-3'UTR WT or FOXO1-3'UTR MUT seed region was amplified by PCR. The transcript was then cloned into a psiCHECK-2 vector(Promega Corporation), downstream of the Renilla luciferase gene. The vector also contained the Firefly luciferase gene. $1 \mathrm{~mL}$ of $293 \mathrm{~T}$ cells $\left(5 \times 10^{4} \mathrm{cells} / \mathrm{mL}\right)$ were seeded into per well of 24 -well plates and co-transfected with $200 \mu \mathrm{L}$ transfection complex, which was mixed of $50 \mu \mathrm{L}$ miR493-5p mimics or miRNA NC, 50 $\mu$ L encoded psiCHECK-2 vectors and 100 $\mu \mathrm{L}$ DMEM/H(Hyclone)diluted Lipofectamine $2000(2 \mu \mathrm{L}$, Invitrogen)for $6 \mathrm{~h}$. The cells were lysed and assayed for luciferase activity at $48 \mathrm{~h}$ post-transfection using a Dual-Luciferase Assay kit(catalog no.RG027; Beyotime Institute of Biotechnology). The firefly luciferase was used as a reference for normalization.

\section{Western Blot analysis}

The tissue or cells were collected and lysed using radioimmunoprecipitation assay buffer(Beyotime Institute of Biotechnology, China)supplemented with PMSF(Beyotime Institute of Biotechnology). The protein concentration was quantified using a BCA kit. A total of 30ug protein was subjected to SDS-PAGE and transferred to polyvinylidene fluoride membrane. Then, the blots were blocked with $5 \%$ fat free milk at room temperature for $1 \mathrm{~h}$ and incubated overnight at 4 with the primary antibody. $\beta$-actin mouse mAb(Bioworld Technology)was used as the control. Blots were then washed and incubated for $1 \mathrm{~h}$ with the appropriate anti-rabbit secondary antibody. Membranes were developed with BeyoECL Plus(Beyotime). 


\section{Measurement of Airway Hyperreactivity}

Mice were anesthetized for the measurement of pulmonary mechanics 24hours after the last OVA challenge. Mice were anesthetized with $50 \mathrm{mg} / \mathrm{kg}$ pentobarbital and instrumented for the measurement of pulmonary mechanics(BUXCO Electronics). Mice were tracheostomized, intubated, and mechanically ventilated at a tidal volume of $0.2 \mathrm{ml}$ and a frequency of $150 \mathrm{breath} / \mathrm{min}$. Lung resistance(RL)was measured in response to increasing doses $(3$ to $20 \mathrm{mg} / \mathrm{ml}$ )of aerosolized acetyl- $\beta$-methylcholine chloride methacholine(Sigma-Aldrich).

\section{H\&E staining}

Mice were killed by anesthesia after the last stimulation, and then their lung tissues were collected, fixed with $10 \%$ formaldehyde solution(Wuxi prospect Chemical Reagent Co., Ltd), embedded in paraffin, and sliced into 5um thickness. The lung tissue sections were immersed in xylene(Sinopharm)for 20 minutes and repeated twice. Then put them into glass cylinders with ethanol(Sinopharm)concentration of 100\%, 95\%, $90 \%, 80 \%$ and $70 \%$ sequently and soak them for 5 minutes. Wash 3 times with $2 \mathrm{ml}$ PBS for 5 minutes each time. After hematoxylin and eosin(Shanghai zhanyun chemical Co.,TLD)staining, neutral resin was used to seal the film, and then it was observed and photographed under inverted microscope(Leica DMI3000B).

\section{Statistical Analysis}

Differences between groups were analyzed by unpaired Student's t-tests or ANOVA(Prism5;GraphPad Software). $P$ value $<0.05$ was considered statistically significant.

\section{RESULTS}

\section{High Throughput Sequencings for aberrant dysregulated miRNA in PBMCs of Asthmatic Children}

Peripheral blood from which PBMCs isolated were collected from asthmatic children, and High throughput sequencing was used to screen different expression of miRNAs. There were 506 miRNAs with fold change $>1.5$ and $\mathrm{P}<0.05$, among which 268 up regulated and 238 down regulated(Fig.1A). GO analysis showed that these aberrant dysregulated miRNAs were related to DNA binding, transcription factor activity, cell inflammation, cell activation, proliferation and so on(Fig.1B). KEEG signal pathway analysis showed that these miRNAs were closely related to Th cell differentiation, and their expression was down regulated in asthmatic children(Fig.1C).

Further, through informatics analysis and published database, 10 miRNAs( 5 up-regulated and 5 downregulated)involved in Th cell differentiation were selected for further study(Fig.1D and 1E). Among them, miR-493-5p attracted our attention due to large differences between groups and small differences within groups, suggesting miR-493-5p may play an important role in Th cell differentiation in asthmatic children.

\section{Down regulation of MiR-493-5p and Higher proportion of Th9 cells in Mice Asthma Models}

We used OVA to induce allergic airway inflammation in mice. The mice were killed by anesthesia after the last stimulation, and then the lung tissues and BALF were collected for the following study. As shown in Fig.2A, IL-9 level in lung tissue and BALF of asthma groups were significantly higher than the control groups. Compared with control groups, the miR-493-5p expression were both obviously diminished in asthma groups(Fig.2B). The marker cytokine of Th9 cells is IL-9, and FOXO1 and IRF4 were reported to be transcription factors that promoted Th9 cell differentiation. As the RT-qPCR data showed in Fig.2C, the IL-9, FOXO1 and IRF4 expression were all significantly upregulated in asthmatic mice. Meanwhile, the Flow cytometry results revealed the higher proportion of CD4 ${ }^{+}$T cells secreting IL-9 in the PBMCs of the asthma mice(Fig.2D). These data suggested that the miR-493-5p was down regulated, but Th9 cell proportion was increased in asthma mice.

\section{MiR-493-5p negatively regulates the differentiation of Th9 cells in vitro}

To investigate the effect of miR-493-5p on Th9 cells differentiation, we isolated CD4 ${ }^{+} \mathrm{T}$ cells from the peripheral blood of normal mice and cultured in vitro as the control group(native T cell group). Then, the cells 
were transfected with miR-493-5p mimic/inhibitor under the condition of inducing Th9 cell differentiation. The RT-qPCR(Fig.3A-C)and Western blot(Fig.3D-G)data showed that the mRNA and protein of FOXO1, IL-9 and IRF4 were downregulated in CD4 ${ }^{+} \mathrm{T}$ cells treated with miR-493-5p mimic and upregulated in $\mathrm{CD}^{+} \mathrm{T}$ cells treated with miR-493-5p inhibitor. Also, the Th9 cells differentiation(by flow cytometry, Fig.3H and 3I)and the change trend of IL-9 level(by ELISA, Fig.3J)was consistent with the proportion of Th9 cells. These results suggested that miR-493-5p could negatively regulate the expression of FOXO1, IL-9 and IRF4 and the differentiation of Th9 cells in vitro.

\section{MiR-493-5p directly targets FOXO1}

As the TargetScan predicted FOXO1 was a target gene of miR-493-5p, we performed the dual luciferase reporter assay to verify the relationship between miR-493-5p and FOXO1. The FOXO1-3'UTR WT or FOXO13'UTR MUT seed region was amplified by PCR, and then cloned into a psiCHECK-2 vector(Promega Corporation), downstream of the Renilla luciferase gene. As Fig.4A showed, compared with in cells co-transfected with FOXO1-3'UTR-WT and miRNA NC, the relative luciferase activity was significantly decreased in cells co-transfected with miR-493-5p mimic and FOXO1-3'UTR-WT. However, the relative luciferase activity didn't change in cells other co-transfected with miR-493-5p mimic and FOXO1-3'UTR MUT or with FOXO1-3'UTR MUT and miRNA NC(Fig.4B), which suggested that miR-493-5p could directly target the 3'UTR of FOXO1 by binding with it.

\section{MiR-493-5p Regulates the Th9 Cells Differentiation by Targeting FOXO1}

Based on the above experimental results that miR-493-5p could negatively regulate the differentiation of Th9 cells in vitro, and directly target the 3'-UTR of FOXO1. We speculated that miR-493-5p regulated the Th9 cells differentiation by targeting FOXO1. To certificate our speculation, we prepared 3 targeted interference sequences for FOXO1(Fig.5A), and the knockdown efficiency of FOXO1 interference sequences was detected by RT-qPCR. The results showed that the FOXO1-1 siRNA was the best interference sequences(Fig.5A), which was used for the following experiments. The isolated CD4 ${ }^{+} \mathrm{T}$ cells were divided into 6 groups(A:Naïve T cell, B:Th9 cell, C: Th9 cell+ mimic NC+FOXO1 NC, D: Th9 cell+ miR-493-5p mimic +FOXO1, E: Th9 cell+ mimic NC+siRNA NC, F: Th9 cell+ miR-493-5p mimic +FOXO1-siRNA), and cells in group B-F were co-transfected with miR-493-5p mimic and FOXO1 overexpressing or siRNA plasmids under the condition of inducing Th9 cell differentiation. Compared with the group E, the mRNA(Fig.5B-D) and protein expression(Fig.5E-H) of FOXO1, IL-9 and IRF4, and IL-9 secretion(Fig.5I) in group F was significantly decreased. Meanwhile, there was no significantly difference between group C and D. The change trend of Th9 cells proportion is parallel to the mRNA and protein expression of FOXO1, IL-9 and IRF4(Fig.5J and $\mathrm{K})$. The results showed that overexpression of FOXO1 in the Th9 cells offset the inhibitory effect of miR493-5p mimic on Th9 cells differentiation, while knockdown FOXO1, the inhibitory effect of miR-493-5p mimic was strengthened. These results indicated that miR-493-5p regulated the Th9 cells differentiation by targeting FOXO1.

\section{MiR-493-5p AgomiR Attenuated Allergic Airway Inflammation and Airway Hyperreactivity}

To investigate the role of miR-493-5p in allergic asthma, we intranasal administered miR-493-5p agomiR or agomiR NC to OVA-immunized mice 3 days before airway challenge with OVA. Four hours after the last challenge, all the mice were killed to collect the lung tissue and BALF. As Fig.6A-C shown, miR-493-5p agomiR treatment significantly reduced the proportion of Th9 cells and the secretion of IL-9 in the lung tissue and BALF, and also significantly suppress the protein expression of FOXO1, IL-9 and IRF4 in lung tissue of OVA-induced asthma mice(Fig.6D-G).

In addition, the HE staining results showed a large number of inflammatory cells infiltrated around the bronchi and blood vessels, inflammatory cells and exudates increased, epithelial cells edema and lumen stenosis were observed in OVA-induced asthma mice. Compared with the OVA-induced asthma mice following with agomiR NC treatment, the inflammatory reaction dramatically attenuated in miR-493-5p agomiR treatment mice(Fig.6H). Furthermore, miR-493-5p agomiR treatment significantly inhibited airway hyperresponsiveness in OVA-induced asthma mice(Fig.6I). These results revealed that miR-493-5p agomiR suppressed the 
inflammation and Th9 cell differentiation in asthmatic mice.

\section{Discussion}

Bronchial asthma in children is a kind of chronic airway inflammation caused by immune regulation disorder(12). In the past, Th1 cells were considered to be the protective factor of bronchial asthma. Among many pathological mechanisms of asthma, the imbalance of Th1/Th2 cells plays a major role, which is considered to be the most classic pathogenesis of asthma $(13,14)$. However, studies have found that the imbalance theory is different from the experimental results. Blocking Th2 cytokines cannot effectively alleviate asthma, so it cannot fully explain the pathological mechanism of bronchial asthma(15). Th9 cell is a new type of CD4 ${ }^{+}$ $\mathrm{T}$ cell subtype, which is named because it mainly secretes IL-9. For a long time, IL-9 has been regarded as a related factor secreted by Th2 cells. Until 2008, Th9 cells were discovered as a new type of CD4 ${ }^{+} \mathrm{T}$ cells(6). Although Th2 cells can also secrete IL-9, the amount of IL-9 secreted by Th2 cells is far less than that of Th9 cells. It was proved that IL-9 in peripheral blood of asthmatic children mainly came from Th9(. Also, studies have further confirmed that the CD4 ${ }^{+} \mathrm{T}$ cells producing IL-9 showed completely different Th cell subtypes, that is Th9: Compared with Th2, Th9 secretes more IL-9, while other cytokines related to Th2, such as IL-4, IL-5 and IL-13, secrete less; Th9 cells did not express GATA binding protein 3(GATA-3), orphan associated receptor(ROR- $\gamma \mathrm{T}$ ) and forkhead box protein P3(Foxp3), the key transcription factors of Th2, Th17 and Treg, respectively(17).

A large number of studies have shown that Th9 is involved in the pathogenesis of asthma. In human asthma, Neurath)et al have found that Th9 and IL-9 in the peripheral blood of adult asthma patients were higher than those of healthy people. IL-9 protein was found in the sputum of asthmatic patients, and the expression of IL-9 increased with the aggravation of the disease(. A similar phenomenon was also found in asthmatic children. Jia et al(found that the levels of IL-9 and IL-4 in peripheral blood of asthmatic children were significantly higher than those of healthy children. In addition, animal experiments showed that not only the proportion of Th9 cells in peripheral blood and the level of IL-9 in asthma model mice were significantly higher than those in control group, but also the level of IL-9 mRNA in lung tissue was significantly increased(20). Our study observed IL-9 level in lung tissue and BALF and the proportion of $\mathrm{CD} 4^{+}$Th9 cells in the PBMCs of asthma group were significantly higher than that of control group, which was consistent with the existing reporters.

Different cytokines can affect the differentiation of primitive $\mathrm{CD} 4^{+} \mathrm{T}$ cells into different subtypes. TGF- $\beta$ and IL-4 can promote Th cells to differentiate into Th9 cells. According to Veldhoen's study, in the absence of IL-6, the synergistic effect of TGF- $\beta 1$ and IL-4 can promote the highly polarized primitive Th cells to differentiate into Th9 cells(. In another experiment, when Dardalhon studied the original CD4 ${ }^{+}$foxp $32^{-} \mathrm{CD}_{62} \mathrm{I}^{+}$ $\mathrm{T}$ cells from mice, he also found that under the combined effect of TGF- $\beta 1$ and IL- 4 , the original cells differentiated into Foxp3-effector T cells that produced IL-9 and IL-10, namely Th9 cells(6). Herein, we successfully induced CD4 ${ }^{+} \mathrm{T}$ cells to differentiate into Th9 cell with TGF- $\beta$, IL- 4 and IFN- $\gamma$. Studies have shown that the development of Th9 and IL-9-producing T cells requires a unique transcription factor, such as IRF4(21), BATF3)and IRF1(23), which have been proved to be transcription factors of IL-9 gene and regulate the transcription of IL-9 gene by binding to the promoter during Th9 cell differentiation. FOXO1 can bind to IL-9 and IRF4 promoters and promote IL-9 production by T cells. Inhibition of FOXO1 can inhibit IL-9 secretion by T cells(11). In this study, we also found significantly upregulated IRF4, FOXO1 and IL-9 in lung tissue of asthma mice, and these mRNA and protein expression were all increased under the condition of inducing Th9 cell differentiation, which was in agreement with the result reported before.

Abnormal miRNA expression has been extensively reported and considered as a marker of human diseases. By searching PubMed, a large number of miRNAs have been reported to be involved in the pathogenesis of asthma(. MiR-493-5p is indicated to be a tumor suppressor gene, but its expression and role in asthma are unknown. In this study, PBMCs were isolated from Peripheral blood of asthmatic children, and high throughput sequencing was used to screen different expression of miRNAs. The informatics analysis results showed that miR-493-5p, which was related to Th cell differentiation, was downregulated in PBMCs of asthmatic children. Further studies showed that the expression of miR-493-5p was downregulated in lung 
tissue and BALF of asthmatic mice, and miR-493-5p could negatively regulate the differentiation of Th9 cells in vitro. Animal experiments have further shown that miR-493-5p AgomiR attenuated allergic airway inflammation and airway hyper reactivity. In addition, using a dual-luciferase reporter system, we have found that FOXO1 is a direct target of miR-493-5p. The subsequent rescue experiments proved miR-493-5p regulating the Th9 cells differentiation by targeting FOXO1.

Actually, our mice models are not obtained through gene editing, but OVA sensitive asthma model. Asthma is a polygenic disease in humans, but in view of ethical issues and current technical limitations, we have only done relevant experiments in model mice, but not in human. In addition, our experiments were carried out to explore the pathological mechanisms of human asthma, and this study might benefit more from some confirmation with human cells. Perhaps there might be a difference between human and mouse cells, which needs our further consummation.

In conclusion, this study demonstrated that miR-493-5p inhibits airway inflammation in asthma through suppressed FOXO1 expression to reduce Th9 cell differentiation, and decreased IL-9 secretion. MiR-4935p/FOXO1 may serve as a potential therapeutic target in the development of asthma.(3486 words)

\section{ACKNOWLEDGEMENTS}

Not applicable.

\section{FUNDING}

This work was supported by the National Natural Science Foundation of China [Zheng-Rong Chen, grant number, 81970027 and 81771676; Yong-Dong Yan, grant number 81870006], Jiangsu Provincial Medical Youth Talent [Zheng-Rong Chen, grant number QNRC2016766], Suzhou Medical Youth Talent [Zheng-Rong Chen, grant number GSWS2019047], science and technology for people's livelihood, Science and Technology Projects of Suzhou sanitary bureau [Yong-Dong Yan, grant number LCZX201409], Key Lab of Respiratory Disease of Suzhou [Chuang-Li Hao, SZS201714].

\section{Availability of data and materials}

All data generated or analyzed during this study are available from the corresponding author on reasonable request.

\section{Author contributions}

WL Zhang, G Li, and HT Dong wrote the main manuscript text and completed the experiments such as cell and molecular biology in vitro and vivo. HM Sun, WJ Gu, XX Zhang and MJ Wang, collected clinical samples. H L, YD Yan, CL Hao and W Ji analyzed clinical data. ZR Chen and CH Zhu are to take responsibility for study design. All authors reviewed the manuscript.

\section{Ethics approval and consent to participate}

This study was approved by the Institutional Ethical Committee of Children's Hospital of Soochow University. A written consent was obtained from all the guardians who participated in this study.

\section{Competing financial interests}

Authors declare no potential conflicts of interest with respect to the authorship and publication of this article.

\section{References}


1. Rothe T, Spagnolo P, Bridevaux P, Clarenbach C, Eich-Wanger C, Meyer F, Miedinger D, Möller A, Nicod L, Nicolet-Chatelain G, Sauty A, Steurer-Stey C, Leuppi J. Diagnosis and Management of Asthma The Swiss Guidelines. Respiration; international review of thoracic diseases2018;95(5):364-80.

2. Pedersen S, Hurd S, Lemanske R, Becker A, Zar H, Sly P, Soto-Quiroz M, Wong G, Bateman E. Global strategy for the diagnosis and management of asthma in children 5 years and younger. Pediatric pulmonology2011;46(1):1-17.

3. Lambrecht B, Hammad H, Fahy J. The Cytokines of Asthma. Immunity2019;50(4):975-91.

4. Coquet J. A singular role for interleukin-9 in the development of asthma. Science immunology2020;5(48).

5. Jia L, Wang Y, Li J, Li S, Zhang Y, Shen J, Tan W, Wu C. Detection of IL-9 producing T cells in the PBMCs of allergic asthmatic patients. BMC immunology2017;18(1):38.

6. Dardalhon V, Awasthi A, Kwon H, Galileos G, Gao W, Sobel R, Mitsdoerffer M, Strom T, Elyaman W, Ho I, Khoury S, Oukka M, Kuchroo V. IL-4 inhibits TGF-beta-induced Foxp3+ T cells and, together with TGF-beta, generates IL-9+ IL-10+ Foxp3(-)effector T cells. Nature immunology2008;9(12):1347-55.

7. Veldhoen M, Uyttenhove C, van Snick J, Helmby H, Westendorf A, Buer J, Martin B, Wilhelm C, Stockinger B. Transforming growth factor-beta 'reprograms' the differentiation of $\mathrm{T}$ helper 2 cells and promotes an interleukin 9-producing subset. Nature immunology2008;9(12):1341-6.

8. Koch S, Sopel N, Finotto S. Th9 and other IL-9-producing cells in allergic asthma. Seminars in immunopathology2017;39(1):55-68.

9. Angkasekwinai P. Th9 Cells in Allergic Disease. Current allergy and asthma reports2019;19(5):29.

10. Lujambio A, Lowe S. The microcosmos of cancer. Nature2012;482(7385):347-55.

11. Buttrick T, Wang W, Yung C, Trieu K, Patel K, Khoury S, Ai X, Elyaman W. Foxo1 Promotes Th9 Cell Differentiation and Airway Allergy. Scientific reports2018;8(1):818.

12. Lloyd C, Saglani S. Opening the Window of Immune Opportunity: Treating Childhood Asthma. Trends in immunology2019;40(9):786-98.

13. Dechene L. TH1/TH2 immune response. The Journal of allergy and clinical immunology2002;110(3):53940; author reply 40.

14. Muehling L, Lawrence M, Woodfolk J. Pathogenic CD4 T cells in patients with asthma. The Journal of allergy and clinical immunology2017;140(6):1523-40.

15. Walker J, McKenzie A. T2 cell development and function. Nature reviews Immunology2018;18(2):121-33.

16. Jia L, Wang Y, Li J, Li S, Zhang Y, Shen J, Tan W, Wu C. Detection of IL-9 producing T cells in the PBMCs of allergic asthmatic patients. BMC Immunol2017 Jul 19;18(1):38.

17. Kaplan M, Hufford M, Olson M. The development and in vivo function of T helper 9 cells. Nature reviews Immunology2015;15(5):295-307.

18. Neurath M, Finotto S. IL-9 signaling as key driver of chronic inflammation in mucosal immunity. Cytokine \& growth factor reviews2016;29:93-9.

19. Hastie AT, Steele C, Dunaway CW, Moore WC, Rector BM, Ampleford E, Li H, Denlinger LC, Jarjour N, Meyers DA, Bleecker ER. Complex association patterns for inflammatory mediators in induced sputum from subjects with asthma. Clinical and experimental allergy : journal of the British Society for Allergy and Clinical Immunology2018 Jul;48(7):787-97.

20. Tong R, Xu L, Liang L, Huang H, Wang R, Zhang Y. Analysis of the levels of Th9 cells and cytokines in the peripheral blood of mice with bronchial asthma. Experimental and therapeutic medicine2018 Mar;15(3):24804. 
21. Staudt V, Bothur E, Klein M, Lingnau K, Reuter S, Grebe N, Gerlitzki B, Hoffmann M, Ulges A, Taube C, Dehzad N, Becker M, Stassen M, Steinborn A, Lohoff M, Schild H, Schmitt E, Bopp T. Interferon-regulatory factor 4 is essential for the developmental program of T helper 9 cells. Immunity2010;33(2):192-202.

22. Lee W, Jang S, Kim H, Kim S, Heo J, Kim G, Lee G. BATF3 is sufficient for the induction of Il9 expression and can compensate for BATF during Th9 cell differentiation. Experimental \& molecular medicine2019;51(11):1-12.

23. Campos Carrascosa L, Klein M, Kitagawa Y, Lückel C, Marini F, König A, Guralnik A, Raifer H, HagnerBenes S, Rädler D, Böck A, Kang C, Lohoff M, Garn H, Schaub B, Berberich-Siebelt F, Sakaguchi S, Bopp T, Huber M. Reciprocal regulation of the I19 locus by counteracting activities of transcription factors IRF1 and IRF4. Nature communications2017;8:15366.

24. Milger K, Götschke J, Krause L, Nathan P, Alessandrini F, Tufman A, Fischer R, Bartel S, Theis F, Behr J, Dehmel S, Mueller N, Kneidinger N, Krauss-Etschmann S. Identification of a plasma miRNA biomarker signature for allergic asthma: A translational approach. Allergy2017;72(12):1962-71.

25. Li J, Panganiban R, Kho A, McGeachie M, Farnam L, Chase R, Weiss S, Lu Q, Tantisira K. Circulating MicroRNAs and Treatment Response in Childhood Asthma. American journal of respiratory and critical care medicine2020;202(1):65-72.

Figures and Legends 


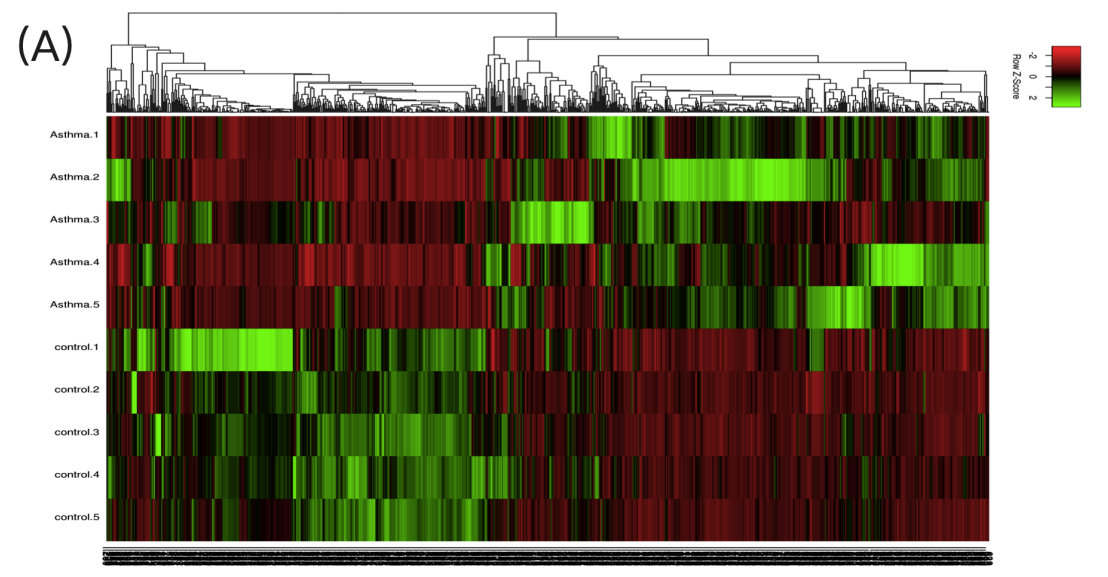

(B)

(1) Up

Sig GO terms of DE gene

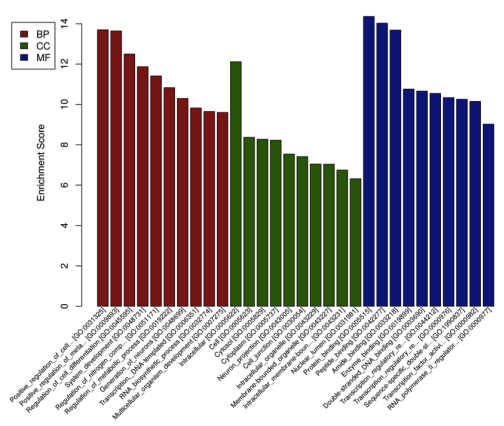

(1) Up

(C)



(2) Down

Sig GO terms of DE gene

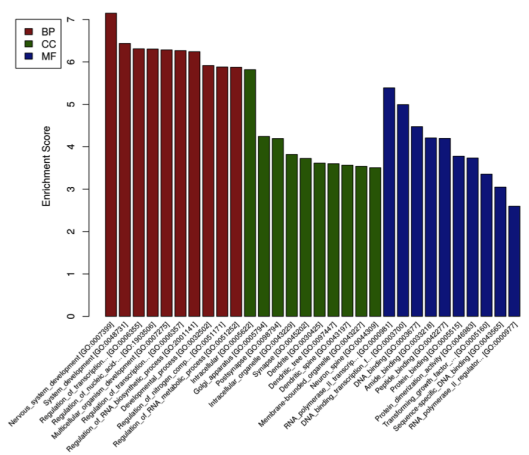

(2) Down

Sig pathway of $D E$ gene

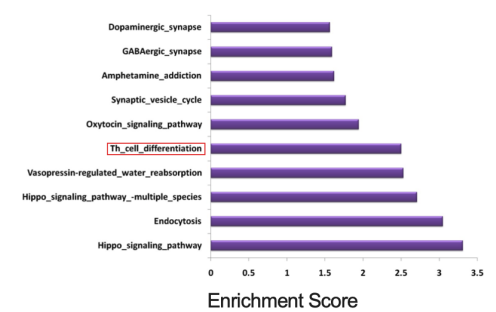

(D)

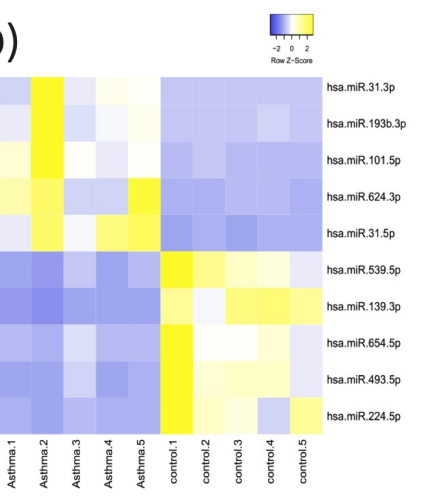

(E)

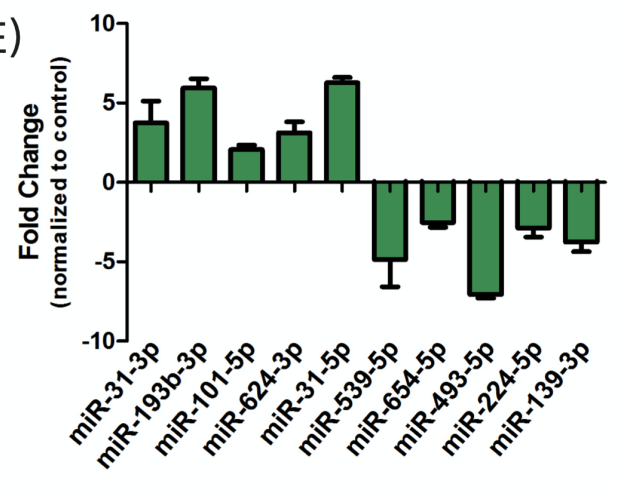


Figure 1: High Throughput Sequencing PBMCs of Asthmatic Children and 10 MiRNAs Related to Th Cell differentiation

Peripheral blood was collected from asthmatic children $(\mathrm{n}=5)$ and normal children $(\mathrm{n}=5)$, and PBMCs were isolated, respectively. And the miRNAs(5 up-regulated and 5 down-regulated)involved in Th cell differentiation were selected for further study.

A. High throughput sequencing was used to screen different expression of miRNAs between asthmatic children and normal children.

B. GO analysis.

C. KEEG signal pathway analysis.

D. Heat map of 10 miRNAs.

E. The fold change of 10 miRNAs.
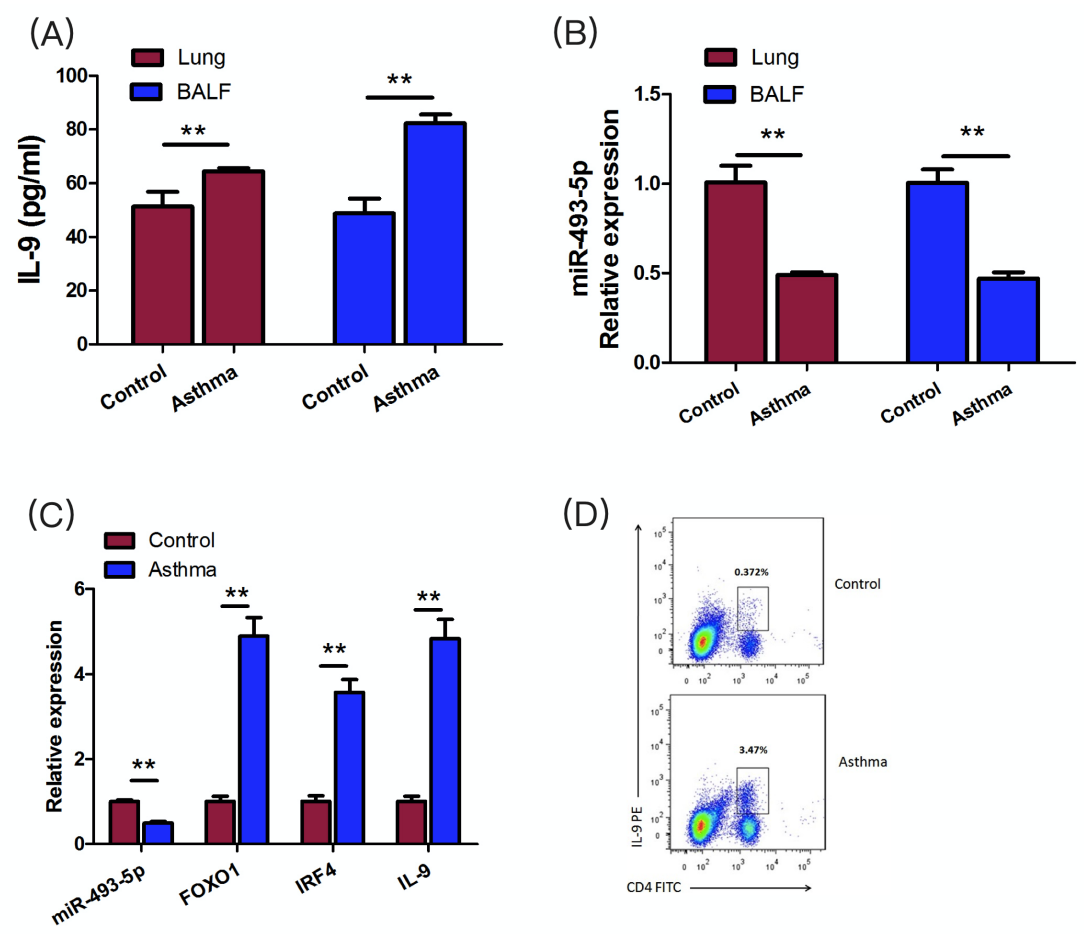

(D)

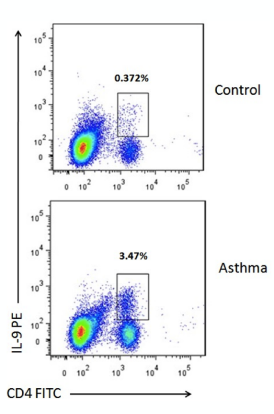

Figure 2: Down regulation of miR-493-5p and Higher proportion of Th9 cells in Murine Asthma Models.

OVA was used to induce allergic airway inflammation in mice $(n=8)$. The mice were killed by anesthesia after the last stimulation, and then their lung tissues and BALF were collected for the following experiments. Untreated mice served as normal controls $(\mathrm{n}=8)$.

A. The IL-9 levels in lung tissue and BALF were measured by ELISA.

B. The miR-493-5p expression in lung tissue and BALF were detected by RT-PCR.

C. FOXO1, IRF4 and IL-9 mRNA expression in lung tissue were detected by RT-PCR.

D. The proportion of $\mathrm{CD} 4^{+} \mathrm{T}$ cells secreting IL-9 in PBMCs were analysed by flow cytometry.

$* * \mathrm{p}<0.01$. 
(A)

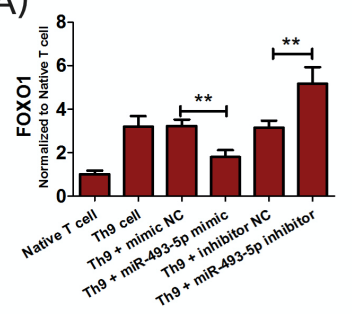

(D) A: Native T cell

B: Th9 cell

C: Th9+mimic NC

D: Th9+miR-493-5P mimic

E: Th9+inhibitor NC

F: Th9+miR-493-5P inhibitor

(E)

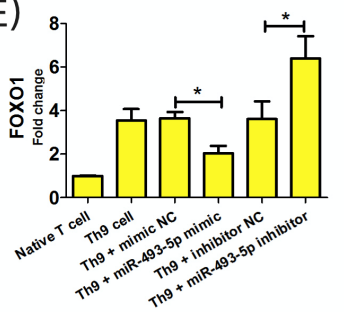

(F)

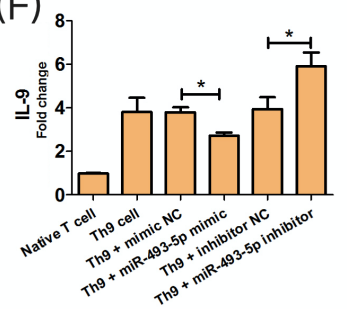

(H)
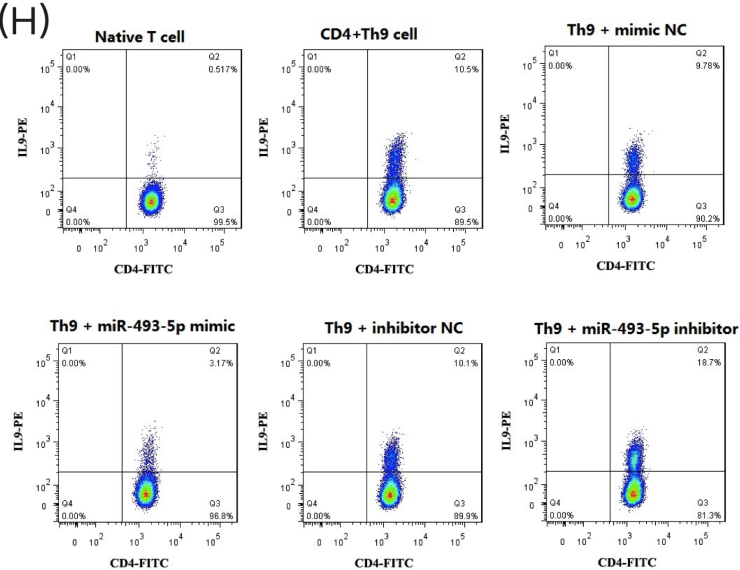

(C)
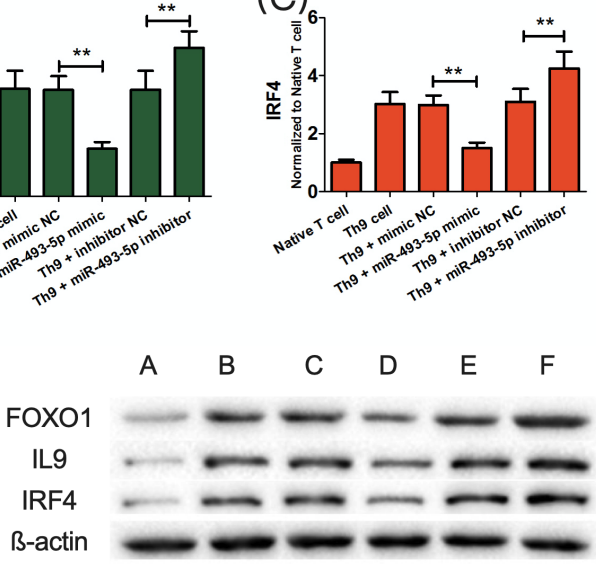

(G)

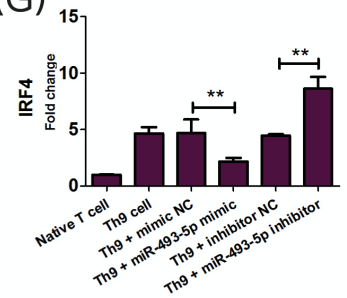

(I)

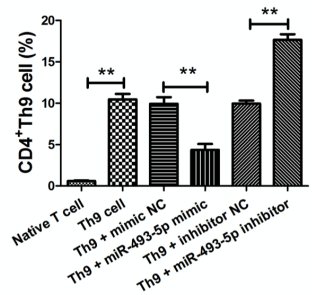

(J)

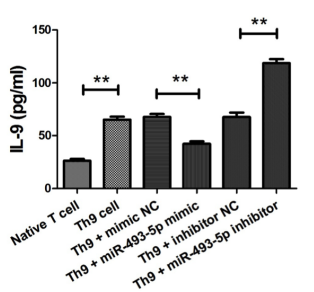

Figure 3: MiR-493-5p negatively regulated the differentiation of Th9 cells in vitro.

The $\mathrm{CD} 4^{+} \mathrm{T}$ cells were separated from normal mice by density gradient centrifugation and magnetic beads. To investigate the effect of miR-493-5p on Th9 Cells differentiation, the CD4 ${ }^{+} \mathrm{T}$ cells were transfected with miR-493-5p mimic/inhibitor and their negative control(NC)under the condition of inducing Th9 cell differentiation.

A-C. The mRNA expression of IL-9, IRF4 and FOXO1 were detected by RT-PCR.

D-G. The protein expression of IL-9, IRF4 and FOXO1 were detected by western blot.

H-I: The proportion of CD4 ${ }^{+}$Th9 cells was analysed by flow cytometry. 
J: IL-9 levels in cell supernatant were measured by ELISA.

${ }^{*} \mathrm{p}<0.05,{ }^{* *} \mathrm{p}<0.01$.

(A)

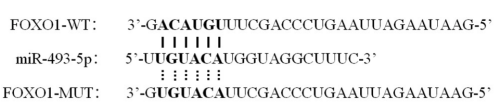

(B)

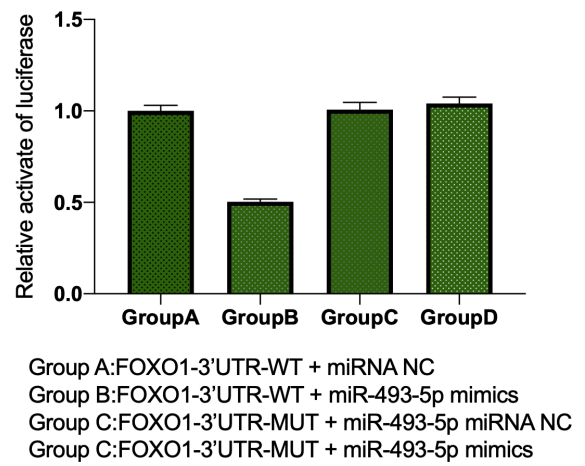

Figure 4: MiR-493-5p directly targets FOXO1

A. The binding sites of miR-493-5p on the 3'UTR of FOXO1, as well as the mutant sites based on TargetScan.

B. Dual luciferase reporter assay was used to assess the luciferase activities of FOXO1 3'UTR WT and FOXO1 3'UTR MUT reporters in cells after miRNA NC or miR-493-5p mimic transfection. 
(A)

FOXO1-siRNA-1 sense: 5'-CCAUGGACAACAACAGUAATT-3' FOXO1-siRNA-2 sense: 5'-GAGCGUGCCCUACUUCAAGTT-3' FOXO1-siRNA-3 sense: 5'-CCAGCUAUAAAUGGACAUUUATT-3'

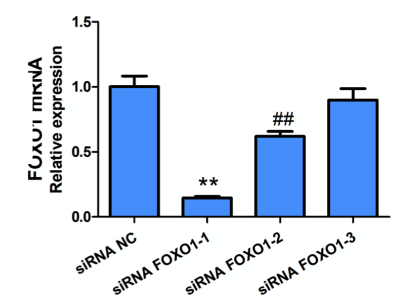

(B)

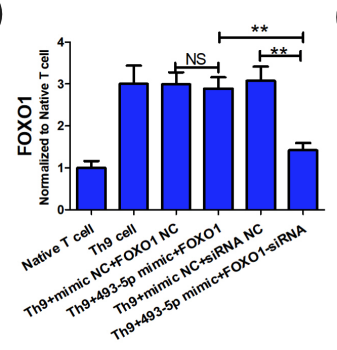

(C)

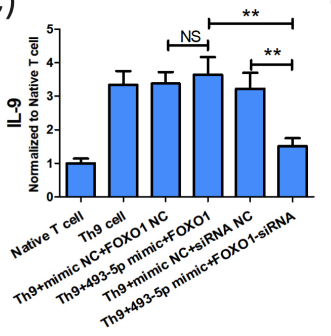

(D)

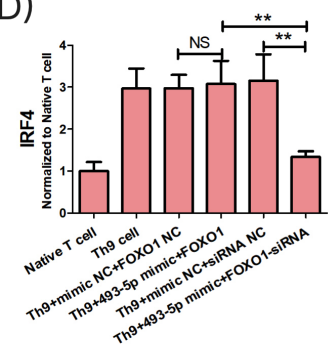

(E)

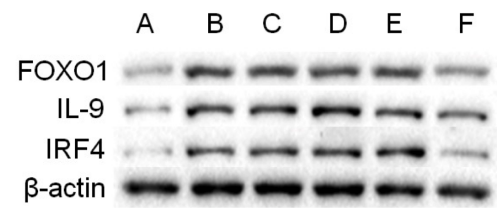

(F)

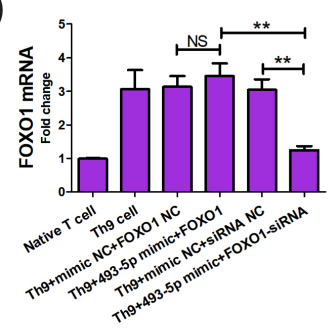

(G)

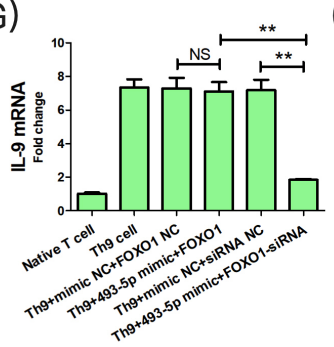

A: Native $C D 4^{+}$ B:CD4+Th9

C:Th9 + mimic NC + FOXO1 NC

D:Th9 + miR-493-5p mimic + FOXO1

$\mathrm{E}:$ Th9 + mimic NC + siRNA NC

F:Th9 + miR-493-5p mimic + FOXO1-siRNA

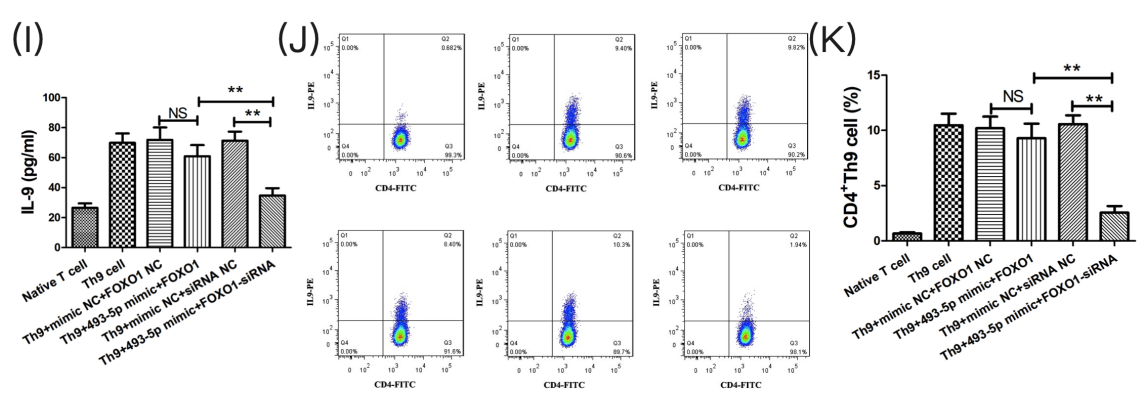

Figure 5: MiR-493-5p Regulates Th9 Cells differentiation by Targeting FOXO1.

To investigate whether miR-493-5p regulates Th9 Cells differentiation by targeting FOXO1, the CD4 ${ }^{+} \mathrm{T}$ cells were co-transfected with miR-493-5p mimic and FOXO1 overexpressing plasmid/interference sequences under the condition of inducing Th9 cell differentiation.

A: Three targeted interference sequences for FOXO1, and comparison of knockout efficiency of FOXO1 interference sequences. 
B-D: The mRNA expression of IL-9, IRF4 and FOXO1 were detected by RT-PCR.

E-H: The protein expression of IL-9, IRF4 and FOXO1 were detected by western blot.

I. IL-9 levels in cell supernatant were measured by ELISA.

J-K: The proportion of CD4 ${ }^{+}$Th9 cells was analysed by flow cytometry.

${ }^{*} \mathrm{p}<0.05,{ }^{* *} \mathrm{p}<0.01, \# \# \mathrm{p}<0.01$.

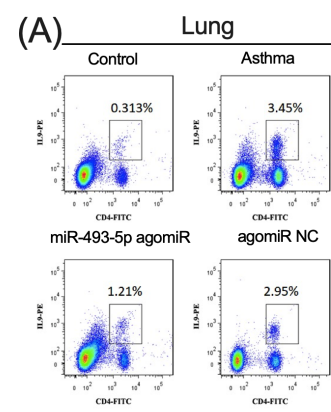

(C)

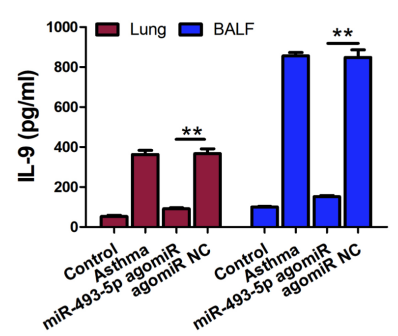

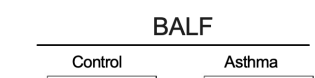

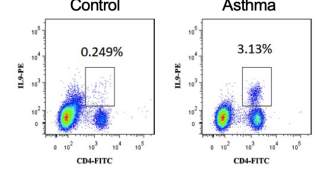

miR-493-5p agomiR $\quad \begin{gathered}\text { cobremic } \\ \text { agomiR NC }\end{gathered}$



(D)

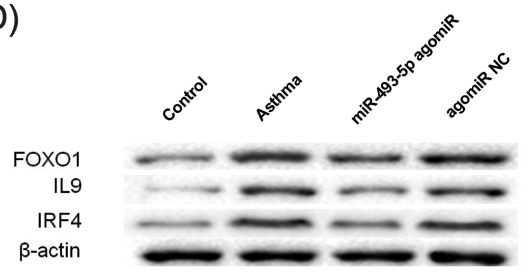

(G)
$(\mathrm{H})$

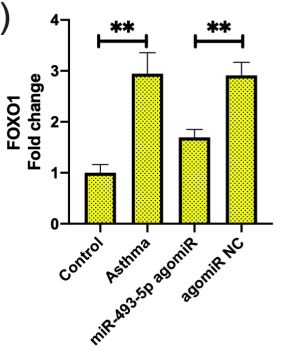

(F)

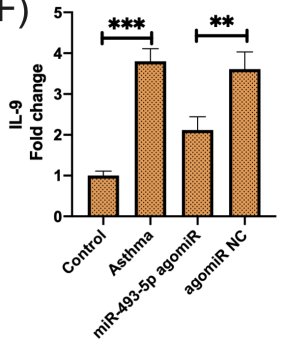

(l)

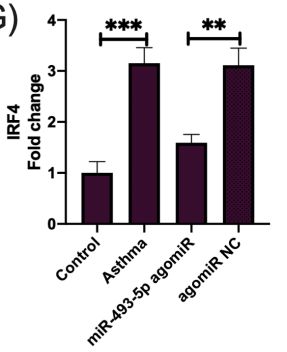

(B)
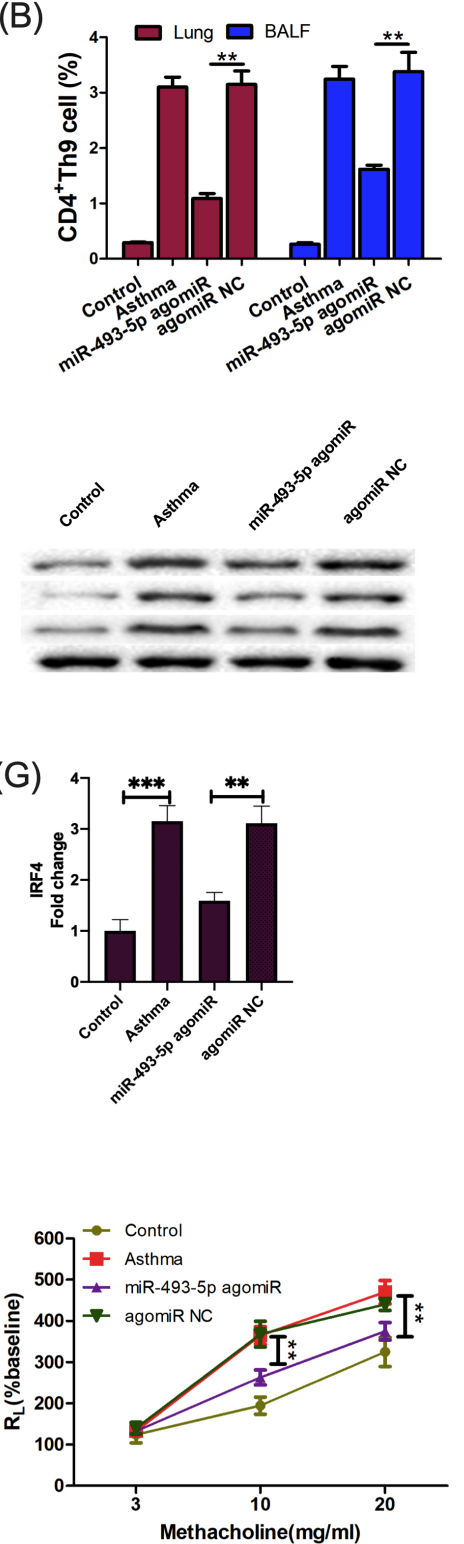

Figure 6: MiR-493-5p AgomiR Attenuates Allergic Airway Inflammation and Airway Hyperreactivity.

BALB/c mice were divided into 4 groups(n=8): Control, asthma, AgomiR NC and MiR-493-5p agomiR. MiR-493-5p agomiR or agomiR NC was given to the asthmatic mice to stimulate miR-493-5p expression before the mice models were stimulated by OVA. 
A-B. The proportion of CD4 ${ }^{+}$T cells secreting IL-9 in lung tissue and BALF were analysed by flow cytometry.

C. IL-9 levels in lung tissue and BALF were measured by ELISA.

D-G. The protein expressions of FOXO1, IRF4 and IL-9 in lung tissue were detected by western blot.

H. The pathological state of lung tissue was evaluated by H\&E staining. Scar bar $=25 \mu \mathrm{m}$.

I. The airway hyperreactivity.

${ }^{*} \mathrm{p}<0.05,{ }^{* *} \mathrm{p}<0.01$.

Table 1. The primer sequences for RT-qPCR

\begin{tabular}{ll}
\hline Primers & Sequences(5'-3') \\
FOXO1-mouse -F & GGGTCCCACAGCAACGATG \\
FOXO1-mouse -R & CACCAGGGAATGCACGTCC \\
IRF4-mouse -F & CTTTGAGGAATTGGTCGAGAGG \\
IRF4-mouse-R & GAGAGCCATAAGGTGCTGTCA \\
IL-9-mouse-F & ACACCGTGCTACAGGGAGG \\
IL-9-mouse-R & TGGTTGCATGGCTTTTCG \\
miR-493-5p-mouse-F & GCCGAGTTGTACATGGTAGG \\
miR-493-5p-mouse-R & CTCAACTGGTGTCGTGGA \\
U6-F & CTCGCTTCGGCAGCACA \\
U6-R & AACGCTTCACGAATTTGCGT \\
$\beta$-actin-mouse-F & GTCCCTCACCCTCCCAAAAG \\
$\beta$-actin-mouse-R & GCTGCCTCAACACCTCAACCC \\
\hline
\end{tabular}

